\title{
Update on Peripherically Inserted Central Catheters in Patients with HIV in a tertiary hospital
}

\section{Lardo $\mathbf{S}^{*}$, Scoppettuolo $\mathbf{G}$ and Dolcetti $\mathbf{L}$}

Department of Infectious Diseases, Agostino Gemelli University Polyclinic, Roma, Rome, Italy

*Corresponding author: Sara Lardo Ph.D, Specialist Doctor, Department of Infectious Diseases, Agostino Gemelli University Polyclinic, Rome, Lazio, Italy, Tel: +989142217299; E-mail: saralardo@gmail.com

Received date: February 6, 2018; Accepted date February 15, 2018; Published date: February 16, 2018

Copyright: (C) 2018 Lardo S, et al. This is an open-access article distributed under the terms of the Creative Commons Attribution License, which permits unrestricted use, distribution, and reproduction in any medium, provided the original author and source are credited.

\begin{abstract}
Literature on patients with HIV and Peripherally Inserted Central Catheter "PICC" published on MEDLINE database in English and Spanish have thoroughly been studied, revised and updated. We reviewed the medical records of all Human Immunodeficiency Virus (HIV) infected patients with Peripherally Inserted Central Catheter "PICC" implanted from 2010 to 2016.

In view of changes over the past in the intravenous management of patients with AIDS and infectious disease, we performed at Policlinico Gemelli Hospital a retrospective study of the last six years, in which we had reviewed all HIV infected patients with PICCs. Retrospectively we have evaluated the clinical severity of patients enrolled using Charlson-Deyo comorbidity score.
\end{abstract}

Our hospital has 1500 beds. There are two departments of infectious diseases for a total of 40 beds. There is an outpatient service where HIV patients are followed and a Day Hospital where chemotherapy is administered.

All HIV- infected patients who had PICC were identified about the occurrence of one of the following events: catheter related complication, catheter requiring removal in all cases, or death. All statistical procedures were performed using the SPSS software package (SPSS Inc. Version 15.0, Chicago).

\section{Introduction}

Ninety two PICCs were inserted in eighty seven patients during the last six years. The indication of placement of PICCs for $40.2 \%$ of patients was intravenous antibiotic therapy, $9.2 \%$ of patients for chemotherapy because of Hodgkin Lymphoma, 20.7\% for NonLymphoma Hodgkin's large cell b, 6.9\% for Cytomegalovirusdisseminated infection, $17.2 \%$ for other cancers, the $4.6 \%$ for Burkitt's lymphoma, the $1.1 \%$ for Kaposi's sarcoma.

PICCs were inserted by use of standard techniques: ECG and XRay techniques. ECG technique was not carried out in $19.5 \%$ of patients (patients who have implanted until 2014), for the other patients (80.5\%) ECG technique was used. In our study it is clear how the technique of implantation of the PICC has changed during these years: from 2014 the use of technologies such as ultrasound and electrocardiographic (ECG) guidance systems to place peripherally inserted central catheters (PICCs) has grown; before 2014 instead it was used X-ray. Among the methods to assess the correct positioning of the tip of central venous catheters, the ECG method is safe and reliable [1].

\section{Discussion}

Pubmed literature shows that PICCs were associated with a low infection rate and a moderate mechanical complication rate $[2,3]$. In our study five of eighty seven patients had a PICCs related complication.
We have defined serious infection as: (1) catheter- related bacteremia-isolation of an organism known to cause catheter-related infections from at least 1 blood culture (or 2 positive blood cultures if the isolate was coagulase-negative Staphylococci) and (2) tunnel infection-tenderness, erythema and/or induration along subcutaneous catheter tract, associated with fever and bacteremia and non-serious infection: (1) exit-site infection-erythema, tenderness, with induration and/or purulence at skin exit site of catheter.

Noninfectious complication: (1) clotted/thrombosis catheter-unable to aspirate or inject via catheter-injection port; (2) leaking or fractured catheter-leaking of catheter due to catheter fracture; (3) catheter migration-catheter fell out or migrated substantially thus requiring removal; and (4) catheter-related phlebitis, local inflammation at the catheter site with a palpable superficial venous cord or induration and serious complication: complication resulting in hospitalization and/or the administration of $i v$ antibiotics $[4,3]$.

The incidence of catheter complications was expressed per 1000 catheter-days. Each catheter was analyzed individually. Among the patients enrolled there are: Three patients with non-serious infection complication, one with thrombosis catheter-unable to aspirate or inject and the two patients because of catheter migration-catheter fell out or migrated requiring removal.

Human immunodeficiency virus (HIV) patients are at risk of developing thrombosis than general population. There are several intersecting mechanisms associated with HIV infection and antiviral therapy that are emerging, which may lead to vasculopathy and hypercoagulability in these patients. 
Citation: Lardo S, Scoppettuolo G and Dolcetti L (2018) Update on Peripherically Inserted Central Catheters in Patients with HIV in a tertiary hospital. J Antimicrob Agents 4: 163. doi:10.4172/2472-1212.1000163

Page 2 of 2

Two patients had serious infection complications: the first one with catheter related bacteremia-isolation of an organism known to cause catheter related infection from at least 1 blood culture (Candida parapsilosis) and the second one with two positive blood culture with coagulase negative Staphylococci (Staphylococcus epidermidis).

In our study thirty-two patients are between the ages of fifty and sixty years, twenty-seven patients in the age group of forty to fifty years; sixty-three male and twenty- four female.
Forty-eight patients had a Charlson-Deyo comorbidity score between 10 and 14 points, sixteen patients a score $>14$ and 23 patients $<10$. The $71.3 \%$ of patients died.

Among the patients enrolled there are: the $72.4 \%$ of patients belongs to the CDC HIV C3 group; CD4 cell counts were $<200$ for thirty-two patients, and $69.6 \%$ of patients had undetectable viremia; the $20.7 \%$ of patients were off therapy and $69 \%$ of patients were treated with antiretroviral therapy (Table 1).

\begin{tabular}{|l|l|l|}
\hline Group & $\mathbf{N}^{\circ}$ patients & $\%$ \\
\hline A1 & 0 & 0 \\
\hline A2 & 2 & $2.30 \%$ \\
\hline A3 & 1 & $1.15 \%$ \\
\hline B1 & 0 & 0 \\
\hline B2 & 9 & $10.34 \%$ \\
\hline B3 & 8 & $9.20 \%$ \\
\hline C1 & 0 & $0.00 \%$ \\
\hline C2 & 4 & $4.60 \%$ \\
\hline C3 & 63 & $72.41 \%$ \\
\hline HIV-RNA $<50 \mathrm{cp} / \mathrm{ml}$ & 64 & $73.56 \%$ \\
\hline HIV-RNA $\geq 50 \mathrm{cp} / \mathrm{ml}$ & 23 & $26.44 \%$ \\
\hline CharlsonScore $\leq 10$ & 31 & $35.63 \%$ \\
\hline $10<$ CharlsonScore<14 & 26 & $29.89 \%$ \\
\hline CharlsonScore $\geq 14$ & 30 & $34.48 \%$ \\
\hline
\end{tabular}

Table 1: Characteristics of patients enrolled in our study

\section{Conclusion}

This study shows that in the last years the risk of patients with Human Immunodeficiency Virus is not the adherence to antiretroviral therapy but the majority of patients with HIV must undergo chemotherapy [5].

PICCs were associated with a low infection rate and a moderate mechanical complication rate, which compare favorably with historical rates seen in AIDS patients with other types of central venous access devices. PICCs are a reasonable alternative to other central venous access devices in patients with HIV or AIDS [4,6].

The use of peripherally inserted central catheter (PICC), with a very low percentage of mechanical and infectious complications, has improved the life expectancy of patients with HIV $[7,8]$.

\section{References}

1. Oliver G, Jones M (2016) ECG-based PICC tip verification system: an evaluation 5 years on. Br J Nurs 25: S4-S10.
2. Mukau L, Talamini MA, Sitzmann JV, Burns RC, McGuire ME (1992) Long-term central venous access vs. other home therapies: Complications in patients with acquired immunodeficiency syndrome. JPEN J Parenter Enteral Nutr 16: 455-459.

3. Skiest DJ, Abbott M, Keiser P (2000) Peripherally inserted central catheters in patients with aids are associated with a low infection rate. Clin Infect Dis 30: 949-952.

4. Hellard M, Fuller A, Spelman D, Spicer WJ, Pickles R, et al. (1997) Totally implantable venous access device infections in patients with AIDS. HIV AIDS 11: 697-698.

5. Goodman HE, Brettle RP, Stevenson B, Hamilton B, Kalima P, et al. (1997) Central venous catheters in patients with AIDS. Int J STD AIDS 8: 417-422.

6. Moore DAJ, Gazzard BG, Nelson MR (1997) Central venous line infections in AIDS. J Infect 34: 35-40.

7. 7. Stanley HD, Charlebois E, Harb G, Jacobson MA (1994) Central venous catheter infections in AIDS patients receiving treatment for cytomegalovirus disease. J Acquir Immune Defic Syndr 7: 272-278.

8. Sweed M, Guenter P, Lucente K, Turner JL, Weingarten MS (1995) Longterm central venous catheters in patients with acquired immunodeficiency syndrome. Am J Infect Control 23: 194-199. 